\title{
The experience of shortening average length of stay in China.
}

\author{
Dong-Cai Li ${ }^{1 \#}$, Han-Rong Cheng ${ }^{2 * \#}$, Wei Zhang ${ }^{1}$, Li Chen ${ }^{1}$, Ai-Wui Weng', Ben-Qing Wu ${ }^{3}$ \\ ${ }^{1}$ Longgang ENT Hospital, Shenzhen, PR China \\ ${ }^{2}$ Shenzhen People's Hospital, Second Clinical Medicine College of Jinan University, Shenzhen, Guangdong, PR China \\ ${ }^{3}$ Children's Medical Center of Guangming New District Medical Group, Shenzhen, Guangdong, PR China \\ \#These authors have contributed equally to this study.
}

\begin{abstract}
Background: The aim of this study was to shorten the average length of stay by using 'Change Acceleration Process'.

Method: We carried out brainstorming with the staff and committee followed by review of questionnaire information, and then intervened with three problems which are of high impact and ease of implementation.

Results: The average length of stay decreased gradually after intervention from 2014 to 2017.

Conclusion: There was potential waste elimination. We shortened the average length of stay while decreasing the rate of unplanned readmission and the proportion of patients hospitalized over $30 \mathrm{~d}$ and improved the patient and staff satisfaction at the same time.
\end{abstract}

Keywords: Average length of stay, Unplanned readmission, Satisfaction, PDSA.

Accepted on August 27, 2018

\section{Introduction}

Average length of stay is an important indicator of hospital service capacity, and is a metric of hospital management and quality of medical care [1]. Shenzhen People's Hospital is an academic medical center with 4195 staff. There are 2300 beds at inpatient department and 99620 admissions in 2016 and over 72000 surgeries in 2016. Increasing health costs is becoming a burden for countries all over the world. It is not solely based on increasing the health spending; every aspect of health expenditure should be considered to improve efficiency. One of our plans is improving efficiency and reducing waste.

PDSA (plan, do, study, action) is rapid-cycle testing process. It is an efficient way to make some improvement to the problem we concerned. We propose a measure to improve efficiency by decreasing the average length of stay. The average length of stay in 2014 is $9.6 \mathrm{~d}$ in Shenzhen People's Hospital, providing less of an incentive for earlier discharge. By reducing the length of stay, there would be significant savings, making healthcare more affordable and sustainable. Shortening the average length of hospital stay can improve the quality of medical care.

\section{Method}

We applied the scientific method 'Change Acceleration Process' to make some improvement in terms of shortening average length of stay (Figure 1).

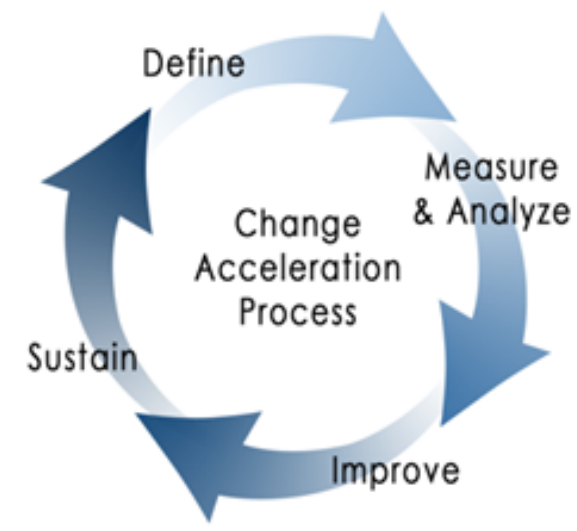

Figure 1. Change acceleration process.

\section{Definition}

Average length of stay is a metric of hospital management and quality of medical care. The standard of hospital service capacity in average length of stay is $9 \mathrm{~d}$, while in our hospital it is $9.8 \mathrm{~d}$ and $9.6 \mathrm{~d}$ during 2013 and 2014 respectively. So our aim was to shorten the average length of stay by using 'Change Acceleration Process'. The application of suitable method and achieving buy in and cooperation of the staff are the key points. Our goals align with internal priorities and help position the institution to meet external mandates. 


\section{Measurement and analysis}

Learning the problem: Establishment of cross-sectoral CQI (continuous quality improvement) group of 10 people, including the director of hospital, quality manager, auxiliary department's leadership and clinical leadership was done. We then carried out brainstorming with the team, designed the questionnaires and analyzed the causes.

Analysis of the causes: We performed brainstorming with frontline staff. 300 questionnaires about average hospital length were distributed, 294 of valid questionnaires were received. In order to analyze LOS objectively, we used the questionnaire as mentioned below (Table 1) that covers all the processes of hospitalized patient. The analysis of causes is shown in Table 2.

Table 1. Questionnaire about impact factor of LOS.

\section{Questionnaire about impact factor of LOS}

1. Do you know the control aim of average LOS in your department?
Yes ( ) No ( )

2. What is the main cause that extends LOS in your opinion?

(1) The progressing disease of patient.

(2) Patients Hospitalized over $30 \mathrm{~d}$.

(3) Waiting for auxiliary examination.

(4) Patient's other reasons.

(5) The doctor is too hands full.

(6) OR room is not enough

(7) Trouble in transferring patient between departments.

(8) Takes time in identifying diagnosis.

(9) Takes time in referring to the other doctor.

(10) Others.

Thank you for your cooperation!

Shenzhen People's Hospital, Jan 2014

Table 2. Statistics showing the causes of prolonged average hospital length according to the questionnaire.

\begin{tabular}{|c|c|c|}
\hline Influencing factors & Was thought to be the main cause & The proportion (\%) \\
\hline Waiting for auxiliary examination & 96 & 32.65 \\
\hline The progressing disease of patient & 76 & 25.85 \\
\hline Patient's other reasons & 63 & 21.43 \\
\hline Trouble in transferring patient between departments & 15 & 5.1 \\
\hline Patients hospitalized over $30 \mathrm{~d}$ & 14 & 4.76 \\
\hline The doctor is too hands full & 12 & 4.08 \\
\hline OR room is not enough & 10 & 3.4 \\
\hline Takes time in identifying diagnosis & 5 & 1.7 \\
\hline Takes time in referring to the other doctor & 3 & 1.02 \\
\hline Total & 294 & 100 \\
\hline
\end{tabular}

Improve: We then intervened three problems which are of high impact and ease of implementation: Reduced duration in waiting auxiliary examination for hospitalized patient; standardized the therapy for various diagnoses; encouraged the frontline staff, clinical leadership and quality manager to participate. In addition, the application for informatics supports all the strategy to be implemented. We applied Plan-Do-StudyAct as rapid-cycle testing.

First intervention: Shorter waiting period of important ancillary examination for hospitalized patients.

Plan: Reduced the waiting time of important auxiliary inspection, encouraged the flexibility arrangement of shift in auxiliary department.

Do: Leadership of radiology department and ultrasound department and gastroenterology department are included. The implementation duration was from January 2015 to December
2016. We got buy in of the staff after they understood the necessity. The staff accepted the flexibility arrangement of shift. The record and the reminder of intranet supported the appointment and its implementation.

Study: The waiting duration of important auxiliary examination including MRI, contrast-enhanced CT, gastrointestinaloscopy, ultrasound for hospitalized patients was shortened, $99 \%$ can be made within three working days of which only $46 \%$ can be done before intervention.

Action: The feedback from clinical department was received by radiology department and ultrasound department and gastroenterology department. Any case whose appointment period was more than three days will be tracked and analyzed. We will keep evaluating every 6 months after the implementation of the project. 
Second intervention: Standardization and homogenization treatment in terms of the patient's serious condition.

Plan: The clinical pathways and single disease was managed by quality department with rewarding measure to clinical staff.

Do: Quality manager and clinical leadership were included. Implementation duration: June 2015-June 2017. Clinical staff and leadership training is the key point to the project. Informatics supported the reporting and monitoring of clinical pathways and single disease cases.

Study: Cases which applied clinical pathways accounted for $67 \%$ of the discharged patients compared to only $20 \%$ of the patients engaged before intervention. This meets the requirement of the hospital management center in China. The reported number of single disease significantly improved. The duration of average hospital stay decreased steadily in the recent four years.

Action: It turned out that the intervention was effective. We will keep long-term management and monitoring of the clinical pathways and single disease, and will keep evaluating every 6 months after the implementation of the project.

Third intervention: Management of patients who were hospitalized over $30 \mathrm{~d}$ and the patient who was readmitted unplanned.

Plan: Patients hospitalized over $30 \mathrm{~d}$ were managed by quality department. We monitored the patient who was readmitted unplanned at the same time.

Do: Quality manager and clinical leadership were involved. Implementation time: June 2015-June 2017. Criteria of access patients hospitalized over $30 \mathrm{~d}$ were set up. The average length of stay, patients hospitalized over $30 \mathrm{~d}$ and the patient who was readmitted unplanned was assessed and monitored every month. The clinical departments and quality manager was requested to analyze these data every 3 months. Incentive policy was applied to encourage the implementation of this project.

Study: The proportion of patients hospitalized over $30 \mathrm{~d}$ compared to discharged patients decreased over the late $3 \mathrm{y}$, and the length of average hospital stay in the hospital decreased.

Action: It showed that the intervention was effective. We will keep evaluating every 6 months after the implementation of the project.

\section{Results}

Data collection, analysis, and report were managed by the Quality Programs and the Decision Support Systems departments.

1. The waiting duration of important auxiliary examination for hospitalized patients was shortened, $99 \%$ of them can be made within three working days whereas only $46 \%$ can be done before intervention.
2. After the management of clinical pathways and single disease, cases which applied for clinical pathways accounted for $67 \%$ of the discharged patients whereas only $20 \%$ of the patients engaged before intervention; so as to standardize and homogenize treatment of patient.

3. The patients hospitalized over $30 \mathrm{~d}$ when compared to discharged patients (\%), declined steadily (Figure 2).

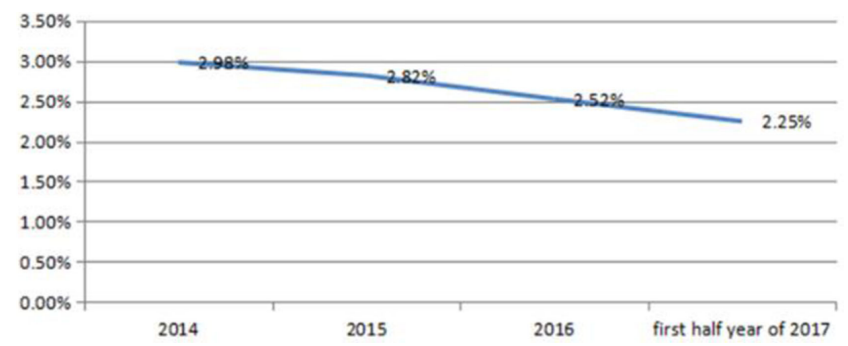

Figure 2. The proportion of patients hospitalized over $30 \mathrm{~d}$ compared to all discharge patients (\%).

4. Unplanned readmission rate of discharged patients within 31 d during 2015-2017 (\%), decreased over time after intervention (Figure 3).

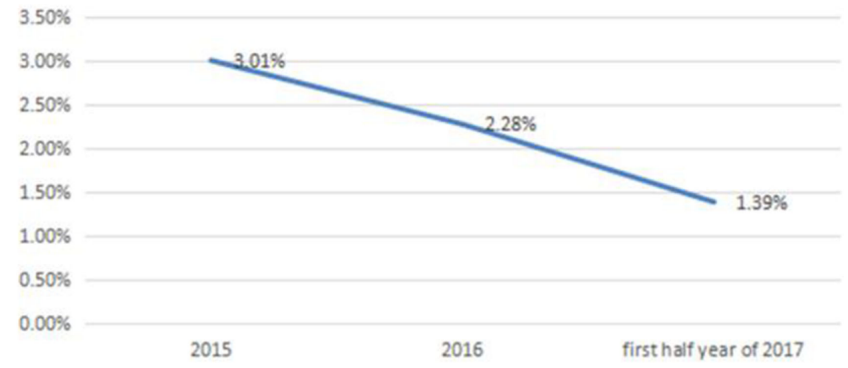

Figure 3. Unplanned readmission rate of discharged patient within 31 d during 2015-2017 (\%).

5. Average length of stay of hospitalised patients over the past 4 y. We can see from the Figure 2, after intervention, the average length of stay of hospitalised patients dropped steadily over time (Figure 4).

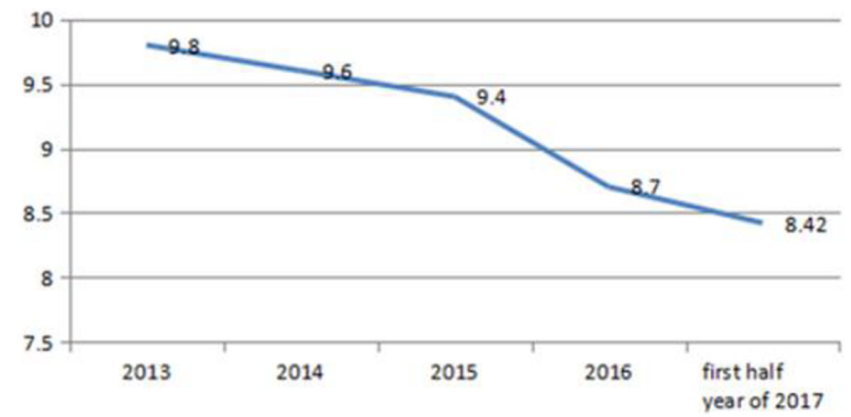

Figure 4. Average length of stay (d).

6. Growing rate of per hospitalization fee was less than the requirement of the hospital management center in China (Figure 5). 


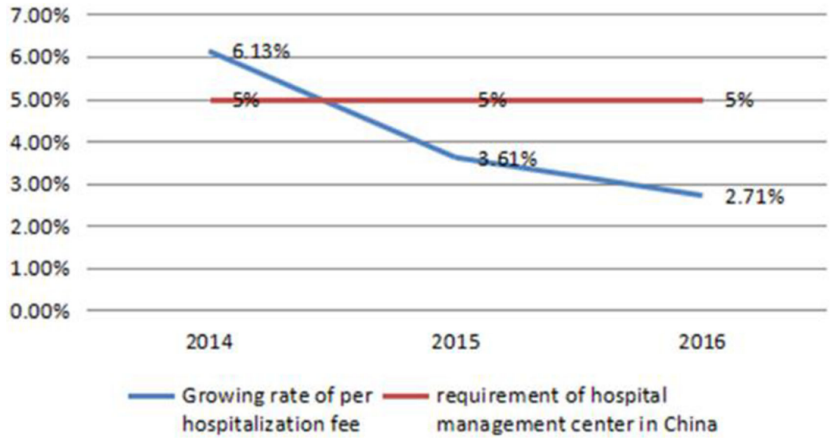

Figure 5. Growing rate of per hospitalization fee in SZ people's hospital.

7. Pre/post design to evaluate inpatient satisfaction, after intervention, patent's satisfaction increased significantly (Table $3)$.

Table 3. Inpatient satisfaction pre/post project implementation.

\begin{tabular}{lll}
\hline Satisfaction survey & Before intervention & After intervention \\
\hline Inpatient & 0.818 & 0.899 \\
\hline
\end{tabular}

\section{Discussion}

The Length of Stay (LOS) in hospitals is the outcome of a complex system of hospital-specific and location-specific factors [2]. The ability to discharge a patient is a key factor in overall LOS and discharge destination is commonly recognised as a major determinant of LOS [3]. The availability of supporting infrastructure such as hospice care, step-down community facilities, nursing homes, GPs per weighted head of population and social care funding will all play a role in delivering lower LOS [4]. The pursuit of lower health care costs is an on-going endeavour and international comparisons are often made against systems with perceived higher efficiency [5-7]. We decreased the average length of stay and the proportion of patient hospitalized over $30 \mathrm{~d}$, while reducing the unplanned readmission. Eventually the growing rate of per hospitalization fee is much lower compared to the requirement of hospital management center in China and inpatient satisfaction improved a lot just like lean which is the relentless pursuit of the perfect process through waste elimination and respect for people.

To achieve this aim, we emphasized three points. First is broad scope and collaboration. Quality work cannot be performed in a silo; it requires broad collaboration with many individuals and across departments. At our hospital, we partnered with the following staff: nursing, clinical providers (faculty and trainees), ancillary staff, information systems, marketing and public affairs, risk management. Second is the leadership engagement and accountability which is essential for success. We employed trained leaders in continuous quality improvement concepts. We involved leaders in the development, selection, and target setting of organizational quality and safety goals. We shared data on an on-going basis with leadership including barriers to success and recommendations for improvement. Last but not the least leaders were accountable for performance of established goals. The third point is health information technology and data. So much of the rest of our lives are going digital and it's high time that health care followed suit. Health information technology (HIT) is an essential part of the solution to increase the efficiency of medical care. HIT is extremely important because of the data that it generates. These data can, for example, make the above-mentioned variability in care transparent and illustrate where there is room for improvement. Conversely, they can also allow us to see where there is brilliance and shines a spotlight on it so others can learn by example. This identification of areas of care that are worrying or exemplary can only really be identified with data. One can't do any of this without data, base lining, and just comparing and then monitoring. Clinical Informatics is a new medical subspecialty for doctors and nurses. Information systems and personal devices will produce vast quantities of data [8].

In the pursuit of efficiency such as length of stay (LOS) comes under increased scrutiny, we take the sustainability into account [9]. The frontline, leadership needs to know about the data. The LOS relevant data is reported out and reviewed monthly. The data is displayed in charts, graphs or dashboards. Implementation of clinical and non-clinical human resource policies that link training, staff education, and performance evaluations to quality and safety goals always include patientcentred care. We standardize works make it easy to do the right thing by fostering standardized work and supporting interventions that will create a physical environment that drives correct workflow, create a trusting environment with close loop feedback and satisfy patient and family needs.

In conclusion, we shorten the average length of stay by using 'Change Acceleration Process'. Incentives on clinical pathways and single diseases should be implemented over time; the incentive measures of reduction of the average hospital day should be implemented. Shortening the ALOS in the long term improves the quality of medical care. This is similar with the study of Zhang which showed that shortening average length of stay improves medical efficiency and benefits hospital [10]. Back to 'define': What's the next improvement opportunity? The next step is: The management of patients hospitalized over 30 days can be taken more measures. Patient and family engagement will contribute to better clinical outcomes. Application of medicare DRG payments will be taken into consideration which is related to length of stay. The last but not the least point is the staff and leadership training in continuous quality improvement concepts.

\section{Acknowledgement}

I would like to my gratitude to Dr Wei Zhang, a respectable scholar, who has provided me with valuable guidance in writing of this paper. 


\section{References}

1. OCDE. Average length of stay in hospitals. Brit J Health Care Management 2013; 19: 186-191.

2. Foer D, Ornstein K, Soriano TA, Kathuria N, Dunn A. Nonmedical factors associated with prolonged hospital length of stay in an urban homebound population. J Hospital Med 2012; 7: 73-78.

3. Elena K, Diana K, Haiyan G. Length of stay as a performance indicator: robust statistical methodology. IMA J Management Mathematics 2005; 16: 369-381.

4. Joon Ho C, Liliana OB, Hway Suh K. Impacts of indoor daylight environments on patient average length of stay (Alos) in a healthcare facility. Building and Environment 2012; 50: 65-75.

5. Qian L, Xinyu Z, Yanan G, Yao Z, Yaxuan W, Bo L, Yaogang W. The correlation of hospital operational efficiency and average length of stay in China: A study based on provincial level data. J Biosci Med 2016; 04: 49-55.

6. Kurtz SM, Lau EC, Ong KL, Adler EM, Kolisek FR, Manley MT. Which clinical and patient factors influence the national economic burden of hospital readmissions after total joint arthroplasty? Clin Orthop Relat Res 2017; 475: 2926-2937.
7. Yao-Jiong X, Meng-Qiu U, Jing Z. Relation between Average length of stay and inpatient's expense. Hospital Management Forum 2016.

8. Simpson S, Packer C, Stevens A, Raftery J. Predicting the impact of new health technologies on average length of stay: development of a prediction framework. Int $\mathrm{J}$ Technol Assess Health Care 2005; 21: 487-491.

9. Veerasingam N, Arany AN. Hospital at home: Sustainable healthcare in developing countries through reducing average length of stay in hospitals. Int J Management Sustainability 2014; 3: 51-61.

10. Wen Xin Z, Bo Heng Z. Shortening average length of stay improves medical efficiency and benefit in hospital. Chinese Health Economics 2007.

\section{*Correspondence to}

Han-Rong Cheng

Shenzhen People's Hospital

Second Clinical Medicine College of Jinan University

Shenzhen

Guangdong

PR China 